\title{
ANÁliSE DO PERFIL CLÍNICO DE CRIANÇAS COM PARALISIA CEREBRAL ATENDIDAS EM UMA CLÍNICA ESCOLA
}

\section{ARTIGO ORIGINAL}

SILVA, Antonio Rafael da ${ }^{1}$

TAVARES, Marcos Raí da Silva ${ }^{2}$

FILGUEIRA, Viviane Gomes Barbosa ${ }^{3}$

SILVA, Antonio Rafael da. TAVARES, Marcos Raí da Silva. FILGUEIRA, Viviane Gomes Barbosa. Análise do perfil clínico de crianças com paralisia cerebral atendidas em uma clínica escola. Revista Científica Multidisciplinar Núcleo do Conhecimento. Ano 05, Ed. 08, Vol. 04, pp. 115-128. Agosto de 2020. ISSN: 24480959, Link de acesso: https://www.nucleodoconhecimento.com.br/saude/perfil-clinico

\section{RESUMO}

A Paralisia Cerebral (PC) é uma patologia decorrente do desenvolvimento anormal do encéfalo, essa desordem no Sistema Nervoso pode ocorrer antes ou após o nascimento, ou até mesmo durante o parto. $O$ objetivo da pesquisa foi descrever o perfil clínico das crianças diagnosticadas com Paralisia Cerebral atendidas em uma clínica escola de fisioterapia. O estudo é do tipo transversal, documental, observacional e com delineamento quantitativo. Foram incluídas no estudo todas as crianças com diagnóstico exclusivo de PC, independente do sexo e idades, admitidas no setor de reabilitação pediátrica. As informações coletadas foram transferidas para um banco de dados por meio do programa Software Microsoft Office Excel 2010. Os

\footnotetext{
${ }^{1}$ Bacharel em Fisioterapia.

2 Pós-graduando Lato Sensu em Fisioterapia Traumato-ortopedia Funcional e Desportiva (Faculdade Inspirar). Graduação em Fisioterapia.

${ }^{3}$ Especialização em docência do ensino superior. Graduação em Fisioterapia.
} 
resultados demonstraram predominâncias dos casos de PC em crianças do sexo masculino. $O$ déficit de oxigênio no cérebro foi à principal causa etiológica e a PC espástica foi à de maior acometimento $\mathrm{A}$ deformidade ortopédica predominante foi a do pé em padrão equino e o dispositivo auxiliar mais utilizado destacou-se a cadeira de rodas. O diagnóstico clínico da PC na maioria dos pacientes foi estabelecido após o primeiro trimestre de desenvolvimento motor. Os comprometimentos mais frequentes estavam o atraso no desenvolvimento neuropsicomotor (ADNPM), seguida da hipertonia, déficit de equilíbrio e coordenação. Dessa forma, o estudo ofereceu a comunidade acadêmica uma descrição do perfil clínico-epidemiológico das crianças com PC atendidas por estagiários de fisioterapia de uma instituição privada de ensino, no interior do estado do Ceará.

Palavras-chave: Paralisia Cerebral, pediatria, fisioterapia.

\section{INTRODUÇÃO}

A paralisia cerebral (PC) ou encefalopatia crônica não progressiva da infância, é uma disfunção neurológica que ocorre no encéfalo em processo de maturação e desenvolvimento, esse comprometimento encefálico pode ocorrer no período pré, peri ou pós-natal (BAX et al., 2005).

A encefalopatia crônica não progressiva da infância acarreta algumas complicações decorrentes da lesão a nível central, dentre elas, pode-se citar: o déficit motor associado às alterações sensoriais, tônus muscular, postura, equilíbrio e coordenação motora (DIAS et al.,2010).

Nos países desenvolvidos as taxas de PC possuem incidência de 1,5 a 5,9 para cada 1000 crianças nascidas vivas e nos países subdesenvolvidos a incidência é de 7 a cada 1000 nascidos vivos (PRIETO et al., 2018). Cerca 70\% dos diagnosticados com PC, são advindos da lesão do neurônio motor superior (GOMES; GOLIN, 2013). 
Nesse contexto, a criança diagnosticada com PC possui padrão de desenvolvimento motor atípico, ou seja, suas habilidades e padrões motores se desenvolvem lentamente e tardiamente devido à lesão cerebral (MADEIRA; CARVALHO, 2009).

A abordagem multidisciplinar na PC deve ser constituída por médicos das diversas especialidades, como também terapeuta ocupacional, fisioterapeuta, fonoaudiólogo, assistente social, educador e psicólogo, visto que, é de suma importância à atuação desses diversos profissionais, visando, reduzir as complicações advindas dessa patologia e consequentemente, promover melhora em sua funcionalidade (CARGNIN; MAZZITELLI, 2003).

A fisioterapia possui função importante na reabilitação da criança com PC, na qual, tem por objetivos inibir reflexos primitivos e tônus anormal, sempre respeitando o desenvolvimento motor típico, a fim, de evitar ou amenizar alterações musculoesqueléticas como aquisições de contraturas e deformidades (OLIVEIRA; GOLIN, 2017).

O objetivo do estudo é investigar o perfil do paciente com Paralisia Cerebral atendido no setor de pediatria Neuroinfantil de em uma clínica escola. Visando oferecer o conhecimento detalhado dos agravos, causas e distribuição da doença na instituição. Em tempo, favorecer o âmbito científico, aumentando o número de pesquisas sobre o tema proposto e que os dados apresentados possam servir como embasamento científico e utilizados em futuros estudos comparativos.

\section{METODOLOGIA}

O presente estudo é do tipo transversal de abordagem documental, de natureza observacional com caráter quantitativo.

A pesquisa foi realizada na Clínica Escola do Centro Universitário Doutor Leão Sampaio, localizada na Av. Maria Letícia Leite Pereira s/n, Campus Lagoa Seca, na Cidade de Juazeiro do Norte-CE. A unidade de estudo ocorreu no setor de Fisioterapia 
Neuroinfantil, onde foi desenvolvida a coleta de dados, durante o mês de Dezembro de 2018.

Foram inclusos no estudo aqueles com o diagnóstico clínico de Paralisia Cerebral e admitido no setor de fisioterapia Neuroinfantil no período de Janeiro a Setembro de 2018. Os critérios de exclusão foram os prontuários daqueles pacientes que não tinham diagnóstico clínico de Paralisa Cerebral e os que apresentaram outra patologia neurológica associada. A desistência do atendimento durante o período estabelecido pelo pesquisador automaticamente o mesmo era excluído do estudo.

A coleta de dados aconteceu em três etapas, no tempo decorrido de duas semanas. Inicialmente ocorreu o levantamento do número de prontuários existentes no setor, contabilizando um universo de 494 fichas/prontuários de avaliações.

$\mathrm{Na}$ segunda fase foi realizada a leitura dos diagnósticos clínicos e automaticamente eram selecionadas as fichas que se enquadrava nos critérios estabelecidos para inclusão da pesquisa, totalizando 91 fichas de avaliações, dessas fichas selecionadas havia documentos duplicados por isso totalizou-se em 91 prontuários.

$\mathrm{Na}$ terceira etapa as informações contidas nas fichas/prontuários foram extraídas e anotadas em um instrumento para elaboração do perfil clínico. Pelo o fato de muitas fichas de avaliação estar preenchidas de forma incompleta, todas tiveram que ser analisadas na tentativa de obter o máximo de informações sobre paciente atendido no setor. Após o levantamento das informações foram incluídos no estudo um total de 14 crianças com diagnóstico clínico de Paralisia cerebral.

As informações coletadas foram transferidas para um banco de dados por meio do programa Software Microsoft Office Excel 2010. O estudo seguiu de acordo com a lei de resolução no 466/12, de 12 de Dezembro de 2012, que regulamenta as pesquisas envolvendo os seres humanos, visando o respeito e proteção desses indivíduos. Esse trabalho envolveu coleta de dados em prontuários e fichas de evoluções dos pacientes de forma retrospectiva, a pesquisa foi submetida à Plataforma Brasil e aprovado pelo o comitê de ética da Instituição de ensino Centro Universitário Doutor Leão Sampaio. 
O presente estudo apresentou riscos mínimos, podendo ocorrer perda e extravio dos prontuários e evoluções, como também, misturas desses documentos e alterações de informações. A fim de evitar esses riscos, os dados foram coletados somente na Clínica Escola, respeitando o horário de funcionamento, onde a análise foi realizada somente pelo pesquisador, de forma cautelosa e mantendo o sigilo pessoal das informações. Os benefícios consistiram em apresentar dados que ainda não tinham sido analisados.

\section{RESULTADOS}

Após a análise dos dados coletados observou-se que $78,6 \%$ das crianças atendidas na clínica escola era do sexo masculino, $21,4 \%$ do sexo feminino com média de idade gestacional de $37,08 \%$ semanas e idade cronológica de aproximadamente sete anos, essas crianças apresentaram um percentual equitativo em relação a partos naturais e cesáreo.

Dentre as possíveis causas etiológicas para o desenvolvimento da Paralisia Cerebral encontradas nos dados dos prontuários destacou-se a hipóxia cerebral correspondendo a $33,3 \%$ dos casos, seguida da prematuridade $22,2 \%$ e icterícia com $16,7 \%$.

Quanto ao tipo de PC verificou-se uma frequência de 11 pacientes que apresentaram sua classificação referente ao tipo de tônus com predominância a do tipo espástica equivalendo a $78,6 \%$. Dentre as deformidades ortopédicas encontradas o pé equino estava em destaque cerca de $75 \%$ apresentaram essa alteração musculoesquelética.

Os únicos dispositivos auxiliares utilizados pelos os pacientes do setor foram à cadeira de rodas e a órtese AFO-Ankle-Foot Orthosis (órtese-tornozelo-pé), e 40\% não utilizavam nenhum tipo de dispositivo.

Outros dados relevantes foram o tempo aproximado para o diagnóstico clínico da PC, onde a maioria das mães recebeu esse parecer médico após o primeiro trimestre de vida da criança. O principal motivo que levaram essas mães a procurarem assistência 
médica, foi devido elas perceberem atraso no desenvolvimento motor de seus filhos, uma vez que, a criança com PC permanece com padrão de desenvolvimento motor atípico persistindo reflexos e padrões, que são comuns no primeiro trimestre da criança, com uma ordem de desenvolvimento totalmente desordenada e não seguindo uma sequência cronológica previsível.

Em relação ao diagnóstico cinético funcional 10 crianças apresentaram atraso no desenvolvimento neuropsicomotor (ADNPM), observa-se que em relação ao diagnóstico topográfico da PC não foi descrito pelo o estagiário impossibilitando a análise topográfica das crianças atendidas no setor.

\section{DISCUSSÕES}

Após a análise da literatura observou-se, que a prevalência global da PC é de 2,11 para cada 1000 nascidos vivos, a faixa de peso entre $1000 \mathrm{~g}$ a $1499 \mathrm{~g}$ com idade gestacional menor que 28 semanas, maiores são as prevalências de PC, divergindo da variável idade gestacional analisada na respectiva pesquisa, onde a média das crianças do setor de Fisioterapia Neuroinfantil foi de 37,08 semanas (OSKOUI et al., 2013).

A hipóxia perinatal ocorre devido a uma inadequada oxigenação para o Sistema Nervoso Central, sendo um tipo de comprometimento de maior ocorrência dentro do processo de reabilitação, correlacionando assim com os resultados do presente estudo (FERREIRA, 2016).

Entretanto os fatores-alvo associado ao início da $\mathrm{PC}$, demonstra que a pré-eclâmpsia era um achado inconsistente ou inconclusivo para o início da lesão. A corioamnionite, o baixo peso ao nascer e a menor idade gestacional mostrou-se como condições favoráveis para o desencadeamento da lesão. Em relação ao gênero, o sexo masculino apresentou achados mistos, mas a prematuridade e o baixo peso era um fator predominante para crianças do gênero masculino ( LIESHOUT et al., 2017). 
A paralisia cerebral espástica foi à de maior ocorrência dentro do setor de Fisioterapia Neuroinfantil da Clínica escola da Unileão, sendo considerado o tipo de lesão encefálica mais comum, estando interligado um quadro clínico de lesão no neurônio motor superior incluindo: hiperreflexia, babinski positivo, hipertonia e fraqueza muscular (GOMES; GOLIN, 2013).

Esse quadro espástico é uma das principais causas das alterações musculoesqueléticas devido à própria modificação do tônus muscular. Dentre as várias modificações podemos destacar: encurtamentos musculares, perda da amplitude de movimento, deformidades como a rotação externa de quadril, flexão de joelhos e pés em padrão equinovaros (RIBEIRO et al., 2017).

Como foi constado na pesquisa que a cadeira de rodas é o dispositivo mais utilizado pelos pacientes, já que, uma parcela significativa necessita desse dispositivo ou algum tipo de carrinhos de transportes, de forma adaptada com assentos específicos. Quanto ao uso adequado da cadeira de rodas na criança com PC, estudos apontam que $80 \%$ utilizam o dispositivo de forma inadequada, dispondo de uma biomecânica estrutural completamente inapropriada, podendo acarretar ou acentuar alterações musculoesqueléticas (CAMPOS, 2013).

Em relação a órtese AFO a maioria das crianças do setor tinha a deformidade do pé com padrão equino, mas não fazia uso do aparelho ortopédico, a utilização de órteses no tornozelo tem bastante eficácia no andar em crianças com PC, esses dispositivos visam o posicionamento adequado das articulações, a fim, de reduzir o reflexo patológico ou a própria espasticidade. Diferentes tipos de órteses podem ser utilizados no paciente com PC e que esses dispositivos proporcionam melhoras nos parâmetros da marcha incluindo amplitude de movimento de joelhos e tornozelos, velocidade, comprimentos das passadas, redução do gasto energético e melhoras da função motora grossa (ABOUTORABI et al., 2017)

O diagnóstico tardio foi um dos dados encontrados na pesquisa, esse fato compromete diretamente na evolução clínica dos pacientes, visto que, uma 
intervenção precoce estará aliada com a neuroplasticidade, podendo amenizar a instalação de alterações musculoesqueléticas.

O tempo para diagnóstico pode ser estabelecido antes dos 5 meses de idade corrigida utilizando-se instrumentos fidedignos para prever o risco de PC, interligada diretamente com a história clínica da criança e da mãe, essas ferramentas incluem: Ressonância Magnética, Avaliação Qualitativa Prechtl de Movimentos Gerais e o Exame Neurológico Infantil de Hammersmith. Para estabelecer o diagnóstico precoce é necessário a monitorização de fatores de riscos detectáveis de recém-nascidos para desencadeamento da PC e o outro fator descrito mesmo sem nenhuma causa etiológica registrada é importante o reconhecimento no atraso nos primeiros marcos motores da criança, geralmente a mãe só percebe após os 5 meses de idade corrigida (NOVAK et al., 2017).

A maioria dos pacientes com paralisia cerebral apresenta atraso no desenvolvimento neuropsicomotor motor (LUNA et al., 2018).

Dentre as inúmeras complicações do paciente, destaca-se alterações motoras, sensoriais, postura, tônus muscular, equilíbrio e coordenação, ressalte-se que as modificações são especificas que irá diferir de acordo com a localização topográfica da lesão (DIAS et al.,2010).

\section{CONSIDERAÇÕES FINAIS}

Os resultados do estudo trouxeram um levantamento detalhado dos pacientes com paralisia cerebral acompanhados por estagiários de fisioterapia do último ano da graduação, oferecendo então a comunidade acadêmica uma descrição do perfilclínico epidemiológico do setor de reabilitação neurológica infantil de uma clínica escola, que atende crianças de uma diversidade de patologias sejam elas neurológicas e/ou ortopédicas.

A pesquisa trouxe questionamentos acerca da abordagem clínica dessas crianças em relação à demora em estabelecer o diagnóstico e a intervenção precoce, isso interfere 
diretamente na evolução do seu quadro clínico e no seu desenvolvimento neuropsicomotor, uma vez que, a abordagem prévia na criança que teve a lesão cerebral é de grande relevância e que deve ser iniciada antes que ocorra a instalação de padrões e posturas anormais, onde a plasticidade neural é ideal para um programa interventivo.

A respeito da utilização das cadeiras de rodas é outro questionamento a ser investigado, quanto ao seu uso por essas crianças e se as mães têm conhecimento das complicações advindas do uso incorreto do dispositivo auxiliar. Observou-se também que apesar da maioria das crianças apresentarem à deformidade do tornozelo em padrão equino, a órtese AFO não era utilizada por grande parte dos pacientes, compete ao estagiário à orientação e prescrição do aparelho ortopédico, juntamente com apoio de uma equipe multidisciplinar.

\section{REFERÊNCIAS}

ABOUTORABI A, et al. Efficacy of ankle foot orthoses types on walking in children with cerebral palsy: A systematic review.Ann Phys Rehabil Med, v.60, n.6, p.393-402, 2017.

BAX, M. et al. Proposeddefinition and classification of cerebral palsy. DevMedChildNeurol, v.47, n.8, p.571-576, 2005.

CAMPOS, M. A. A. D. Cadeira de rodas e acessórios para adequação postural na paralisia cerebral: Uma análise documental. Cadernos Brasileiros de Terapia Ocupacional, v. 21, n.1, p.43-49, 2013.

CARGNIN, A. P. M.; MAZZITELLI, C. Proposta de Tratamento Fisioterapêutico para Crianças Portadoras de Paralisia Cerebral Espástica, com Ênfase nas Alterações Musculoesqueléticas. Revista Neurociências, v. 11, p. 34-9, 2003.

DIAS, A. C. B. et al. Desempenho funcional de crianças com paralisia cerebral participantes de tratamento multidisciplinar. Rev. Fisioterapia Pesquisa, Paulo - SP, v.17, n.3, p.225-229, 2010. 
EKIZ, Timur et al. Wheelchair appropriateness in children with cerebral palsy: A single center experience. Journal of back and musculoskeletal rehabilitation, v. 30 , n. 4 , p. 825-828, 2017.

FERREIRA, M. T. S. Incidência de crianças com paralisia cerebral atendidas na clínica escola de fisioterapia da unicatólica. Revista expressão católica. v.1, n.1, 2016.

GOMES, C. O.; GOLIN, M.O. Tratamento fisioterapêutico na paralisia cerebral tetraparesia espástica, segundo conceito Bobath. Revista Neurociência.v.21, n.2, p.278-285, 2013.

LIESHOUT, V.P.; CANDUNDO, H.; MARTINO, R.; SHIN, S.; BARAKAT-HADDAD, C. Onset factors in cerebral palsy: a systematic review. Neurotoxicology, v. 61, p. 4753, 2017.

LUNA, M.M.A.;LUCENA, P.L.; FARIAS, A.E.M.; MELO, V.F.C. O acompanhamento fisioterapêutico de crianças com sequelas de paralisia cerebral atendidas no NASF do município de Alagoa Nova. Revista de Pesquisa: Cuidado é Fundamental Online, v. 10 , n. 3, p. $70-73,2018$.

MADEIRA, E. A. A.; CARVALHO, S. G. Paralisia Cerebral e fatores de risco ao desenvolvimento motor: uma revisão teórica. Cadernos de Pós-Graduação em Distúrbios do Desenvolvimento, São Paulo, v.9, n.1, p.142-163, 2009.

NOVAK, lona et al. Early, accurate diagnosis and early intervention in cerebral palsy: advances in diagnosis and treatment. JAMA pediatrics, v. 171, n. 9, p. 897-907, 2017.

OLIVEIRA, L. S. O.; GOLIN, M. R. Técnica para redução do tônus e alongamento Muscular passivo: efeitos na amplitude de movimento de crianças com Paralisia Cerebral espástica. ABCS Health Sci. v.42, n.1, p.27-33, 2017.

OSKOUI, Maryam et al. An update on the prevalence of cerebral palsy: a systematic review and meta-analysis. Developmental Medicine \& Child Neurology, v. 55, n. 6, p. 509-519, 2013. 
PRIETO, A. V. et al. equoterapia na reabilitação de indivíduos com paralisia cerebral: uma revisão sistemática de ensaios clínicos. Cadernos Brasileiros de Terapia Ocupacional v. 26, n. 1, p. 207-218, 2018.

RIBEIRO, D. S. et al. Alterações musculoesqueléticas em crianças com paralisia cerebral no município de Jequié-Bahia. Ciência \& Desenvolvimento-Revista Eletrônica da FAINOR. v.10, n.1, 2017.

Enviado: Maio, 2020.

Aprovado: Agosto, 2020. 